JOURNAL DE PHYSIQUE

Colloque C3, Supplément au $\mathrm{n}^{\circ} 9$, Tome 49, septembre 1988

\title{
MODELISATION DE L'IMPACT DE PLAQUES MINCES PAR DES PROJECTILES DE PETITS CALIBRES METHODE INVERSE
}

\author{
L. PENAZZI* * * et N. DAHAN* * \\ "Etablissement Technique Central de I'Armement, CREA, 16 bis. \\ Avenue Prieur-de-la-Côte-d'Or, F-94114 Arcueil Cedex, France \\ * Ecole Normale Supérieure de Cachan, Laboratoire de Mécanique \\ et Technologie, 61, Avenue du Président Wilson, F-94230 Cachan. \\ France
}

Résumé:

La résolution du problème de l'impact à faible vitesse ( 100 à $300 \mathrm{~m} / \mathrm{s})$, d'un projectile sphérique rigide sur des plaques minces métalliques,est calculé par une méthode inverse. Nous utilisons la théorie convective en grandes déformations et nous faisons l'hypothèse d'un comportement rigide plastique écrovissable de la plaque. Le bilan énergétique pendant le contact permet d'accéder, de manière analytique, à toutes les informations utiles. Un critère de rupture locale, de type énergétique à été introduit pour prévoir la perforation. Des expériences ont été faites sur l'alliage léger A-G5 ( 5096), les aciers E 24 (A 283 -C) et Z6CND18/12 (316) pour accéder à l'identification des paramètres du champ de déplacements, par une méthode cinématographique ultrarapide.

\begin{abstract}
:
The resolution of low speed impact problem ( 100 to $300 \mathrm{~m} / \mathrm{s})$ created by rigid spherical target on thin metallic is solved by an inverse method. We use the convective theory in large deformations and make plate hardenig rigid plastic comportement hypothesis. The energetic balance during contact, permit to access by analytical method, at alls usefull informations. A local criterium rupture (energetical type), is injected to allow the perforation. Some experimentations have been made on light 5096 alloy, low carbon AISI A 283-C steel, austenitic 316 steel to have access to the identification of displacement field parameters by an ultra-speed cinematographic method.
\end{abstract}

\section{Introduction}

Le problème dynamique des impacts n'est pas récent. De nombreux auteurs J.A. ZUKAS 11/. AVERBUCH et BODNER /2/, M. RAVID /3/ se sont interessés aux problèmes d'impacts sur des plaques épaisses, N. CRITESCU /4/, N.LEVY et W. GOLDSMITH /5//6/, entre autres, ont abordé le problème de la modèlisation de l'impact sur plaques minces . Très souvent ce problème à été considéré comme un problème incrémental en force, et l'on a cherché à résoudre les équations d'équilibre de la dynamique. Nous proposons une modèlisation de type mécanique des milieux continus, en faisant le choix d'un champ de placements cinématiquement admissible. L'équation du bilan énergétique de l'ensemble bille-plaque pendant la duree du contact, dans un cas isotherme ( N. DAHAN /7/) et l'hypothèse, pour la gamme de vitesses étudiées, d'un comportement Rigide Plastique Ecrouissable du matériau de la plaque permettent d'accéder à l'énergie mécanique dissipée par la déformation $W_{p 2}$, à l'énergie cinétique de la bille $K_{1}$ et de la plaque $K_{2}$ pendant le contact. Le choix d'un critère locale d'énergie de rupture ductile, rend compte de la localisation de la déformation et donne une information sur l'énergie résiduelle du projectile. En notant $W_{a 2}$ l'énergie absorbée par la plaque à chaque étape du contact, on peut écrire :

$$
\left.W_{\mathrm{a} 2}(t)=W_{\mathrm{p} 2}(t)+K_{2}(t)+K_{1}(0)-K_{1}(t) \quad \forall t \in\right] 0, t_{f}[
$$


La figure 1 montre pour trois matériaux différents l'évolution de l'énergie absorbée en fonction de la vitesse d'impact. Dans la gamme de vitesses étudiées expérimentalement, pour l'A-G5 et l'acier E 24, on atteint la vitesse limite de perforation, pour laquelle l'énergie de déformation plastique est maximale. Cette limite est associée à la ductilité du matériau car pour l'acier austénitique 316, ce seuil n'a pas été atteint. Nous pouvons comparer sur la figure 2 les différences de comportement en traction statique. Les valeurs extrêmes des courbes correspondant au point d'instabilité plastique.

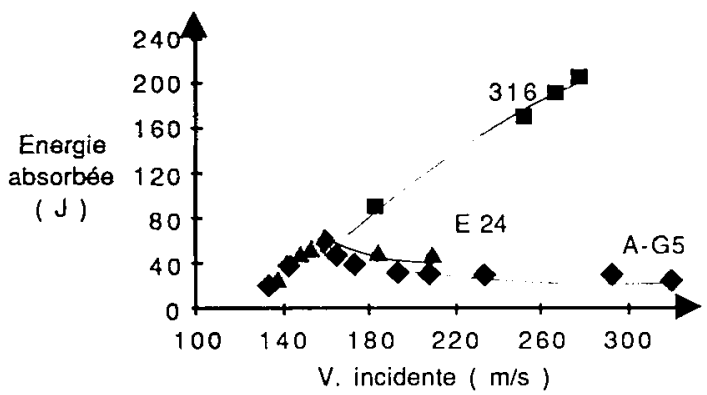

Figure 1 : Evolution de l'energie absorbée par la plaque en fonction de la vitesse incidente.

Plate absorbed energy evolution versus incident speed $(e=1 \mathrm{~mm}, \mathrm{~d}=11 \mathrm{~mm})$.

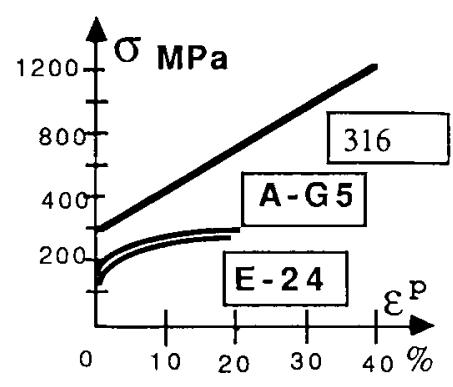

Figure 2 : Traction statique. Static traction.

\section{2- Modélisation}

\section{2-1 Choix des paramètres}

Nous supposons que toute évolution pourrait être considérée comme une succession d'états d'équilibre, c'est à dire que le temps de réponse de la matière est inférieure au temps de propagation du phénomène. Nous choisissons comme variables d'états observables :

- Ie déplacement axial ou enfoncement $U_{t}(0, t)$

- la déformation $\varepsilon(M o, t)$

Le système se comporte d'un point de vue symbolique comme une "boite noire" dans laquelle est introduit une énergie cinétique du projectile $K_{1}$, et de laquelle il ressort les énergies cinétiques $\mathrm{K}_{1}, \mathrm{~K}_{2}$ énergie cinétique de la plaque et la dissipation mécanique de la plaque $W_{2}$, le projectile étant supposé indéformable .

\section{2-2 Bilan d'énergie}

On isole la bille (1) et la plaque (2) ;la conservation de l'énergie bille-plaque à chaque instant du contact s'écrit :

$$
\frac{M}{2}\left[v_{1}^{2}(t)-v_{1}^{2}(0)\right]+\frac{1}{2} \int_{D_{2}} \rho \overrightarrow{v(t)} \cdot \overrightarrow{v(t)} d v+\int_{0}^{t}\left[\int_{D_{2}} \sigma: D d v\right] d t=0
$$

En introduisant $U_{\mathfrak{t}}, \dot{U}_{\mathfrak{t}}$ respectivement l'enfoncement axial et la vitesse d'enfoncement axial et en faisant apparaitre $\mathbb{D}^{*}=\frac{\partial \mathbb{D}}{\partial U_{t}}$ et $\quad \overrightarrow{V^{*}}=\frac{\partial \overrightarrow{V^{*}}}{\partial U_{t}}$

[2] devient : 


$$
M_{1} v_{10}^{2}=\left[M_{1}+\int_{D_{2}} \rho_{2} \cdot \vec{v}^{*} \cdot \vec{v}^{*} \cdot d v\right] \cdot \dot{U} t^{2}+2 \cdot \int_{0}^{t}\left[\int_{D_{2}} \sigma: \mathbb{D}^{*} \cdot U t \cdot d v\right] d t
$$

qui est la nouvelle expression du bilan energétique dans laquelle tous les termes ne dépendent plus que de l'enfoncement $U_{t}$, qui est une variable observable .

\section{2-3 Champ de déplacements}

Nous utilisons les hypothèses suivantes : - décomposition de la deformée de la face intérieure $(\hat{\rho}, \hat{z})$ et la matière dans l'épaisseur $\left(\rho^{*}, z^{*}\right)$

Dans le repère axisymétrique $(0, \vec{r}, \vec{\theta}, \vec{z})$ :

- conservation Incale du volume.

$$
\begin{aligned}
& \vec{U}\left(r_{0}, z_{0}, U_{t}\right)=\rho\left(r_{0}, z_{0}, U_{t}\right) \vec{r}+z\left(r_{0}, z_{0}, U_{t}\right) \vec{z} \\
& \text { avec } \rho\left(r_{0}, z_{0}, U_{t}\right)=\hat{\rho}\left(r_{0}, U_{t}\right)+\rho^{*}\left(r_{0}, z_{0}, U_{t}\right) \\
& z\left(r_{0}, z_{0}, U_{t}\right)=\hat{z}\left(\rho, U_{t}\right)+z^{*}\left(r_{0}, z_{0}, U_{t}\right)
\end{aligned}
$$

Parmi les Champs Cinématiquement Admissible, nous choisissons :

$\hat{z}\left(r, U_{t}\right)=U_{t} \cdot \exp \left[-H \cdot\left[\frac{\hat{\rho}}{R_{0}-\hat{\rho}}\right]\right] ; \hat{\rho}\left(r_{0}, U_{t}\right)=\left\{\begin{array}{rr}r_{0} \cdot\left(k \cdot U_{t} 2\left(R_{0}-r_{0}\right)+1\right) \\ 0 r\end{array}\right.$

où $H, L, k, R_{0}$ sont des paramètres à identifier

\section{2-4 Quantités cinématiques E. $\dot{E}$}

L'utilisation de la théorie convective, de nature Lagrangienne pour l'expression des dẹformations et des vitesses de déformations permet d'accéder à l'expression des quantités $\mathbf{E}$ , E sur le repère local $S_{0}$, associé à la configuration initiale, de manière à pouvoir directement utiliser les lois de comportement du matériau .

D'après N.DAHAN /7/,L.PENAZZ1/8/, le champ des déformations et le champ des vitesses de déformations font intervenir respectivement la métrique $W$ et sa vitesse $W$ :

$$
\begin{aligned}
& E=\left(S_{0}-1\right)^{t} \cdot W \cdot S_{0}-1 \quad \text { avec } W=\frac{1}{2} \cdot\left(G_{t}-G_{0}\right) \\
& \dot{E}=\left(S_{0}-1\right)^{t} \cdot \dot{W} \cdot S_{0}-1
\end{aligned}
$$

\section{2-5 Loi de comportement}

Le comportement du matériau étant assimilable au cas Bigide Plastique Ecrouissable, nous prenons une expression de type LUDWICK :

$$
\bar{\sigma}=\sigma_{\mathbf{y o}}+\mathbf{K} \bar{\varepsilon}^{\mathbf{n}}
$$

\section{2-6 Vitesse d'enfoncement}

En tenant compte de la loi de comportement du matériau, et en utilisant l'équation du

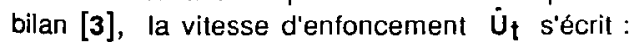




$$
\dot{U}^{2}=\frac{M_{1} \cdot v_{10}^{2}-2 \cdot \int_{D_{2}} w_{p 2}\left(M_{0}\right) \cdot d v}{M_{1}+\int_{D_{2}} \rho_{2} \cdot\left[\left(\frac{\partial \rho}{\partial U t}\right)^{2}+\left(\frac{\partial z}{\partial U t}\right)^{2}\right] d v} ; w_{p 2}=\left[\sigma_{y 0}+\frac{K}{n+1} \cdot \bar{\varepsilon}\left(M_{0}\right)^{n}\right] \cdot \bar{\varepsilon}\left(M_{0}\right)
$$

\section{2-7 Critère de rupture local}

II existe principalement deux approches pour prédire l'instabilité ou la rupture :

- l'une prend en compte la microstructure du matériau en particulier la morphologie inclusionnaire : RICE et TRACEY, THOMASON, MC CLINTOCK ...

- l'autre ne fait intervenir que des grandeurs mécaniques macroscopiques, par exemple l'energie de déformation plastique SIH/9/,'endommagement KATCHANOV,

Nous choisissons un critère basé sur une limite locale de l'énergie de déformation plastique $110 /$. Pour un enfoncement donné $U_{t}$ minimal l'ensemble des points de fracture sera :

$$
c^{f}=\left\{M_{0} \subset D_{2} / U_{t} \operatorname{mini} w_{p 2}\left(M_{0}, \widetilde{U}_{t}\right)=w_{p} f \quad \frac{\partial w_{p 2}-}{\partial M_{0}}=0 \text { et } \frac{\partial^{2}-w_{p 2}-}{\partial M_{0}^{2}}<0\right.
$$

II en résulte : $\quad \frac{\partial w_{\mathrm{R} 2-}}{\partial M_{\mathrm{O}}}=0 \quad \Leftrightarrow \quad \frac{\partial \bar{\varepsilon}}{\partial M_{0}}=0$,

$$
w_{p 2}\left(M_{0}, U_{t}\right)=w_{p} \quad \Leftrightarrow \bar{\varepsilon}\left(M_{0}, \tilde{U}_{t}\right)=\bar{\varepsilon}_{c} \quad \tilde{U}_{t}=\inf \left(U_{t}\right)
$$

\section{3- Analyse expérimentale}

\section{3-1 Présentation des movens}

Les études ont été réalisées sur un lanceur à gaz. L'instrumentation utilisée se composait: - de microbases optiques pour les mesures de vitesses incidente et résiduelles de la bille et du bouchon,

- d'une caméra électronique IMACON équipée soit de tiroirs à images intégrales ( pour le suivi de l'évolution des profils de la plaque), soit de tiroirs à balayage de fente (pour la mesure des déplacements de la bille et de plaque) ( Figures 3 et 4 ),

- de ponts d'extensométrie à haute bande passante ( $f c>75 \mathrm{KHz}$ ) pour des enregistrements de signaux de jauges de déformations placées sur les plaques (BEAUMONT /11/). 


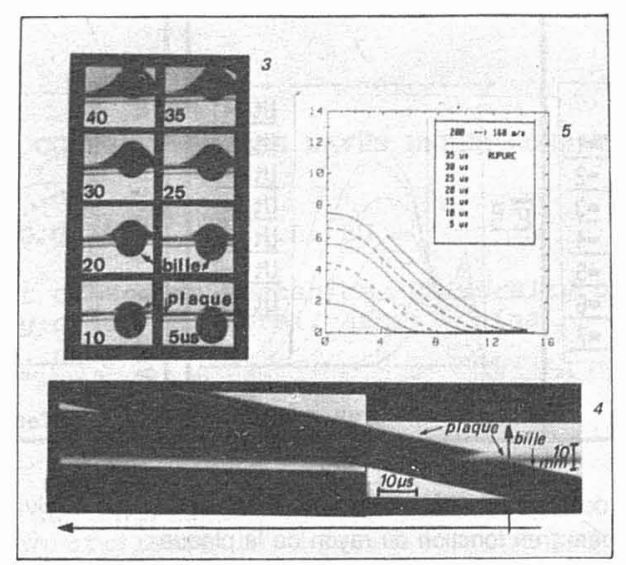

Eigures 3 et 4 : Enregistrements d'un impact sur caméra rapide électronique . Impact recordings by electronic speed camera

Figure 5 : Exemple de digitalisation de profil de déformée de plaque au cours de l'impact. Example of plate distording profile digitalisation during impact .

\section{3-2 Utilisation des informations}

Les relevés cinématographiques par images intégrales permettent d'accéder à l'évolution de la zone de déflection. En suivant le déplacement par rapport au rayon, d'un point d'ordonnée fixée $\mathbf{M}_{\mathrm{L}}(\mathrm{rL}, \mathbf{z} \mathrm{L})$ ( figure 5 ), où $\mathbf{z}_{\mathrm{L}} \ll \mathrm{U}_{\mathrm{t}}$, on s'aperçoit que rapidement après le début du contact $t>2 \mu \mathrm{s}$ ( sinon pour $t \leq 2 \mu \mathrm{s}$ l'ensemble des déplacements de la plaque peut être calculé par les équations de la mécanique des ondes ), la vitesse de déplacement de $M$ L peut s'exprimer par la relation :

$$
\mathrm{Vr}_{\mathbf{L}}{ }^{2}=\mathrm{K} /\left(\mathbf{2} \cdot \mathrm{p}_{\mathbf{2}} \cdot(\mathbf{1}+\mathrm{v})\right)
$$

où $v$ est le coefficient de Poisson, $\rho_{2}$ masse volumique de la plaque

$$
\mathrm{K} \text { la pente d'écrouissage (ct. [12]) (figure 2) }
$$

Les images à fente permettent d'accéder à l'évolution des vitesses de la bille et de la plaque au cours de l'impact (figure 7).

\section{4- Simulation numérique}

A partir de cette analyse, nous avons simulé, sur un calculateur H.P. série 200/300, le cas d'un impact à $200 \mathrm{~m} / \mathrm{s}$, d'une bille d'acier $100 \mathrm{C} 6 \quad(\mathrm{~d}=11 \mathrm{~mm}$, masse $=5.5 \mathrm{~g}$.) sur une plaque d'A-G5 de $1 \mathrm{~mm}$ d'épaisseur. Les mesures cinématographiques permettent l'identification des paramètres :

$k=10^{-6}$

$L=2$ car le projectile est sphérique

$H=R_{0}^{2} /(2$. Ut.$R)$ conditions aux limites $\partial^{2} z(0) / \partial r_{0}^{2}=0(R:$ rayon de la

$\mathbf{R}_{\mathbf{O}}=\mathrm{r}_{\mathrm{L}} \cdot\left(1+\operatorname{Ln}\left(\mathrm{U}_{\mathrm{t}} / \mathrm{zL}\right) / \mathrm{H}\right)$

bille)

$\bar{\varepsilon}_{c}=.20$ Déformation équivalente au point d'instabilité en traction (figure 2)

Nous présentons sur les figures 6 et 7 , l'évolution de l'épaisseur de la plaque et de la déformation plastique équivalente à différentes étapes de l'enfoncement. Nous donnons aussi sur la figure 8 une comparaison de l'évolution de la vitesse d'enfoncement calculée et expérimentale. 

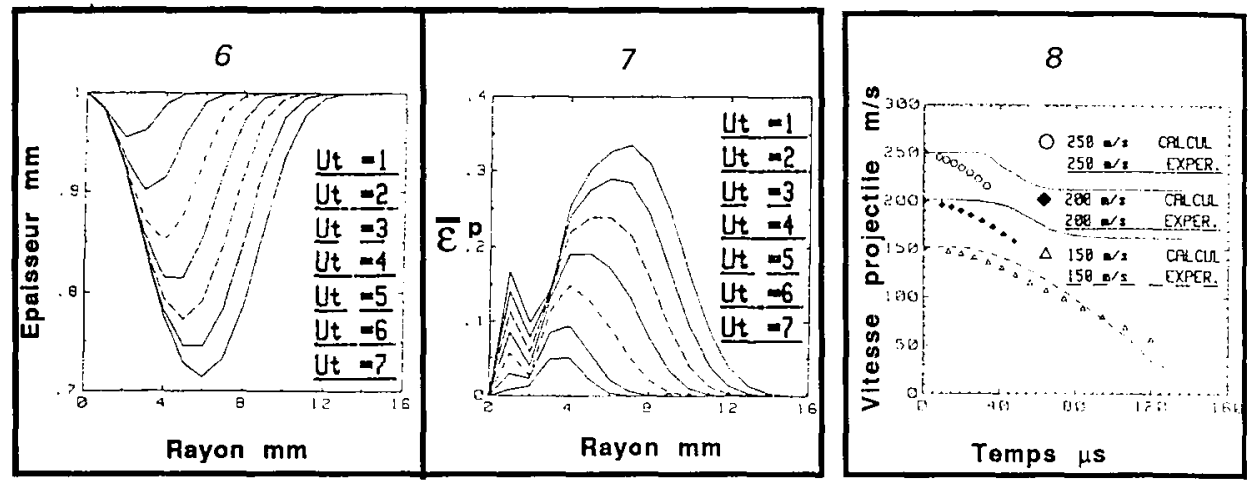

Eigures 6 et 7 : Evolutions de l'épaisseur et de la déformation plastique équivalente au cours de l'enfoncement, en fonction du rayon de la plaque.

Thicknèss and equivalent plastic strain evolution during staving, versus plate radius .

Eiqure 8: Evolutions de la vitesse du projectile au cours de l'enfoncement, pour différentes vitesses d'impacts en fonction du temps (simulation et expérience) Target speed evolution during staving, at different impacts speeds versus time (simulation and experimentation).

\section{5- Discussion des résultats}

Nous avons remarqué que sur des essais statiques de traction, la déformation, dans la zone d'apparition de l'instabilité plastique, était du même ordre de grandeur que celle située dans la zone de localisation de la striction, sur la plaque.

La simulation semble en bonne accord avec l'expérience. Les différences que l'on peut observer sur l'évolution de la vitesse du projectile, entre l'expérience et le calcul , proviennent du choix trop simpliste de la loi de comportement. En effet une loi du type Rigide Plastique Ecrouissable, ne prenant donc pas en compte l'influence de la vitesse de sollicitation, maximise la dissipation plastique. Néammoins les vitesses de déformations sont suffisamment élevées pour que des phénomènes d'adoucissement thermique interviennent par le biais d'une augmentation de la ductilité du matériau et des effets d'inertie .

\section{6- Bibliographie}

$/ 1 /$ J.A. ZUKAS, T.NICHOLAS, H.F. SWIFT, L.B. GRESZCZUK, D.R. CURRAN, Interscience Publications, John Whiley \& Sons Inc., New York 1982

12/ J. AWERBUCK, S.R. BODNER, Int. J. Solids Structures, V 10, 671-684, 1974

/3/ M. RAVID, Communication privée Creusot-Loire, 7/05/1986

/4/ N. CRITESCU, North-Holland publishing compagny, Amsterdam 1967

15/ W.GOLDSMITH, London Edward Arnold Ltd, 1960

$16 /$ N. LEVY et W.GOLDSMITH, Int. J. of Imp. Eng., V2, N3,209-229,1984

$17 /$ N. DAHAN, Thèse de Dr Ing., Univ. Paris VI, 20/12/1979

18/ L.PENAZZI, mémoire de D.E.A , Univ. Paris VI, 24/06/85

$19 /$ N. DAHAN, L. PENAZZI, Colloque EUROMECH 217, Budapest HONGRIE, 1986

$110 /$ G.C. SIH, Int. J. Fracture Mech.,5,365-377,1983

$/ 11 /$ N. BEAUMONT, Colloque AMAC , Paris, 1988 